\title{
Novel potential predictive markers of sunitinib outcomes in long-term responders versus primary refractory patients with metastatic clear-cell renal cell carcinoma
}

\author{
Javier Puente ${ }^{1}$, Nuria Laínez ${ }^{2}$, Marta Dueñas ${ }^{3,4}$, María José Méndez-Vidal' ${ }^{5}$, Emilio \\ Esteban $^{6}$, Daniel Castellano ${ }^{4,7}$, Mónica Martinez-Fernández ${ }^{3,4}$, Laura Basterretxea ${ }^{8}$, \\ María José Juan-Fita ${ }^{9}$, Luis Antón ${ }^{10}$, Luis León ${ }^{11}$, Julio Lambea ${ }^{12}$, Begoña Pérez- \\ Valderrama ${ }^{13}$, Sergio Vázquez ${ }^{14}$, Cristina Suarez ${ }^{15}$, Xavier Garcia del Muro ${ }^{16}$, Enrique \\ Gallardo ${ }^{17}$, José Pablo Maroto ${ }^{18}$, M Luz Samaniego ${ }^{19}$, Beatriz Suárez-Paniagua ${ }^{20}$, \\ Julián Sanz ${ }^{21}$, Jesús M. Paramio ${ }^{3,4}$, SOGUG (Spanish Oncology Genitourinary Group) \\ ${ }^{1}$ Medical Oncology Department, Instituto de Investigación Biomédica, Hospital Clínico Universitario San Carlos, Madrid, Spain \\ ${ }^{2}$ Medical Oncology Department, Complejo Hospitalario de Navarra, Pamplona, Spain \\ ${ }^{3}$ Molecular Oncology Unit CIEMAT and Instituto Investigación Biomédica, Hospital Universitario 12 de Octubre, Madrid, Spain \\ ${ }^{4}$ CIBERONC, Spain \\ ${ }^{5}$ Medical Oncology Department, Hospital Universitario Reina Sofía, Córdoba, Spain \\ ${ }^{6}$ Medical Oncology Department, Hospital Universitario Central de Asturias, Oviedo, Spain \\ ${ }^{7}$ Medical Oncology Department, and Instituto Investigación Biomédica, Hospital Universitario 12 de Octubre, Madrid, Spain \\ ${ }^{8}$ Medical Oncology Department, Hospital Donostia, Donostia, Spain \\ ${ }^{9}$ Medical Oncology Department, Instituto Valenciano de Oncología, Valencia, Spain \\ ${ }^{10}$ Medical Oncology Department, Complejo Hospitalario Universitario de A Coruña, A Coruña, Spain \\ ${ }^{11}$ Promoción e Planificación da Investigación Sanitaria, Axencia de Coñecemento en Saúde, Santiago de Compostela, Spain \\ ${ }^{12}$ Medical Oncology Department, Hospital Clínico de Zaragoza, Zaragoza, Spain \\ ${ }^{13}$ Medical Oncology Department, Hospital Universitario Virgen del Rocío, Sevilla, Spain \\ ${ }^{14}$ Medical Oncology Department, Hospital Universitario Lucus Augusti, Lugo, Spain \\ ${ }^{15}$ Vall d'Hebron Institute of Oncology, Hospital Universitari Vall d' Hebron, Universitat Autònoma de Barcelona, Barcelona, Spain \\ ${ }^{16}$ Medical Oncology Department, Institut Català d'Oncologia, Hospital Duran i Reynals, L'Hospitalet, Barcelona, Spain \\ ${ }^{17}$ Medical Oncology Department, Hospital Universitari Parc Taulí, Sabadell, Spain \\ ${ }^{18}$ Medical Oncology Department, Hospital de la Santa Creu i Sant Pau, Barcelona, Spain \\ ${ }^{19}$ Statistical Department, Trial Form Support TFS people, Madrid, Spain \\ ${ }^{20}$ Oncology Medical Department, Trial Form Support, Madrid, Spain \\ ${ }^{21}$ Pathology Department, Hospital Clínico Universitario San Carlos, Madrid, Spain \\ Correspondence to: Javier Puente, email: javier.puente@ salud.madrid.org \\ Keywords: sunitinib, metastatic renal cell carcinoma, biomarkers, long-term responders, primary refractory \\ Received: December 22, $2016 \quad$ Accepted: February 24, $2017 \quad$ Published: March 23, 2017 \\ Copyright: Puente et al. This is an open-access article distributed under the terms of the Creative Commons Attribution License (CC-BY), \\ which permits unrestricted use, distribution, and reproduction in any medium, provided the original author and source are credited.
}

\section{ABSTRACT}

Background: Several potential predictive markers of efficacy of targeted agents in patients with metastatic renal cell carcinoma ( $\mathrm{MRCC}$ ) have been identified. Interindividual heterogeneity warrants further investigation.

Patients and methods: Multicenter, observational, retrospective study in patients with clear-cell mRCC treated with sunitinib. Patients were classified in two groups: long-term responders (LR) (progression-free survival (PFS) $\geq 22$ months and at least stable disease), and primary refractory (PR) (progressive disease within 3-months of sunitinib onset). Objectives were to compare baseline clinical factors in 
both populations and to correlate tumor expression of selected signaling pathways components with sunitinib PFS.

Results: 123 patients were analyzed (97 LR, 26 PR). In the LR cohort, overall response rate was $79 \%$ and median duration of best response was $\mathbf{3 0}$ months. Median PFS and overall survival were 43.2 (95\% confidence intervals[CI]:37.2-49.3) and 63.5 months (95\%CI:55.1-71.9), respectively. At baseline PR patients had a significantly lower proportion of nephrectomies, higher lactate dehydrogenase and platelets levels, lower hemoglobin, shorter time to and higher presence of metastases, and increased Fuhrman grade. Higher levels of HEYL, HEY and HES1 were observed in LR, although only HEYL discriminated populations significantly (AUC $[R O C]=0.704$; cut-off=34.85). Increased levels of hsa-miR-27b, hsa-miR-23b and hsa-miR-628-5p were also associated with prolonged survival. No statistical significant associations between hsa-miR-23b or hsa-miR-27b and the expression of c-Met were found.

Conclusions: Certain mRCC patients treated with sunitinib achieve extremely long-term responses. Favorable baseline hematology values and longer time to metastasis may predict longer PFS. HEYL, hsa-miR-27b, hsa-miR-23b and hsa-miR628-5p could be potentially used as biomarkers of sunitinib response.

\section{INTRODUCTION}

Over the past years multiple molecularly targeted agents for the treatment of advanced renal cell carcinoma (RCC) have been developed, greatly improving clinical benefit to patients [1].

Sunitinib, a multitargeted tyrosine kinase inhibitor, was approved worldwide as a first-line treatment for selected clear-cell metastatic RCC (mRCC) patients. Sunitinib efficacy was established in a pivotal Phase 3 study comparing sunitinib and interferon- $\alpha$ as firstline treatment in 750 patients with $\mathrm{mRCC}$, showing a significantly higher objective response ratio ( $47 \%$ vs $12 \%$, respectively; $P<0.001$ ), significantly longer progressionfree survival (PFS) (median: 11 vs 5 months; Hazard ratio[HR $]=0.539 ; 95 \%$ confidence intervals $[\mathrm{CI}]: 0.451$, $0.643 ; P<0.001)$, and longer overall survival (OS) (median: 26.4 vs 21.8 months; HR=0.82; 95\% CI:0.67, $1.00, P=0.051)$ in the sunitinib group [2]. However, $9-21 \%$ of patients treated with sunitinib exhibit progressive disease as best response.

Similar to other conventional and moleculartargeted antitumor agents, there is an interindividual variability in response to sunitinib treatment. Potential serum-, radiological-, clinical- and tissue-based predictive biomarkers have been investigated across multiple agents [3-5], obtaining promising results. Additionally, certain adverse events (AEs) (i.e. Hypertension, Hypothyroidism) induced by sunitinib in $\mathrm{mRCC}$ patients may be associated with an improvement in clinical outcomes $[6,7]$.

Inherited genetic variability may be one of several contributing tumor- and host-related factors underlying individual treatment response. Specific signaling pathways such as Notch [8], Hedgehog [9] and Wingless (Wnt)$\beta$-catenin [10] are critical for embryonic development, and for self-renewal and differentiation in adult stem cells, being also involved in tumor development and maintenance [11]. Remarkably, they are also involved in normal and pathological angiogenesis and, thus, have been considered as indicators of response to specific antiangiogenic therapies.

The microRNAs (miRNAs) are key regulators of oncogenic processes [12], representing a promising novel group of biomarkers. They are essential modulators of the above commented developmental signaling pathways, and are also involved in cancer stem cell maintenance [13].

The efficacy and safety of sunitinib in a subset of long-term responders has been previously described in retrospective pooled cohorts [14-16], although a comparison of extreme responders and non-responders is unpublished.

In the present analysis, we compared the baseline clinical characteristics and the levels of expression of various genes and miRNAs involved in Notch, Hedgehog, Wnt, hypoxia, epithelial mesenchymal transition and stem cell maintenance signaling in two extreme groups of patients: those with the highest benefit (long-term responders, LR) and those resistant to sunitinib (primary refractory, PR). We also explored the occurrence of several AEs as potential biomarkers of sunitinib efficacy.

\section{RESULTS}

\section{Clinical characteristics}

One hundred and twenty-three patients from 16 Spanish centers were included, of which 97 (79\%) were LR and 26 (21\%) were identified as PR. In the LR cohort, partial response was achieved in $59 \%$ of patients, complete response in $21 \%$ of patients and SD in $21 \%$. Median duration of best response was 29 months and 33 months in partial and complete responders, respectively. At the time of analysis 51 patients had progressed. Median PFS was 43.2 months (95\%CI: 37.2-49.3). Median OS was 63.5 
Table 1: Demographic and clinical characteristics

\begin{tabular}{|c|c|c|c|c|}
\hline \multicolumn{2}{|l|}{$\begin{array}{l}\text { Baseline patient } \\
\text { characteristics }\end{array}$} & \multirow{2}{*}{$\frac{\mathbf{L R}(\mathbf{n}=\mathbf{9 7})}{44(55.0)}$} & \multirow{2}{*}{$\frac{\operatorname{PR}(\mathbf{n}=\mathbf{2 6})}{5(23.8)}$} & \multirow{2}{*}{$\begin{array}{c}\boldsymbol{P} \text {-value } \\
<0.01\end{array}$} \\
\hline ECOG PS, $N(\%)$ & 0 & & & \\
\hline & 1 & $34(42.5)$ & $13(61.9)$ & \\
\hline & 2 & $0(0)$ & $3(14.3)$ & \\
\hline \multirow[t]{2}{*}{ Nephrectomy, $N(\%)$} & No & $2(2.1)$ & $4(15.4)$ & $<0.01$ \\
\hline & Yes & $94(97.9)$ & $22(84.6)$ & \\
\hline \multicolumn{2}{|l|}{ LDH, UI/L, mean $(95 \% \mathrm{CI})$} & $\begin{array}{c}258.4 \\
(235.9-281.0)\end{array}$ & $\begin{array}{c}380.3 \\
(317.1-443.6)\end{array}$ & $<0.001$ \\
\hline \multicolumn{2}{|c|}{ Hemoglobin, g/dl, mean $(95 \% \mathrm{CI})$} & $\begin{array}{c}13.81 \\
(13.46-14.17)\end{array}$ & $\begin{array}{c}11.96 \\
(11.07-12.86)\end{array}$ & $<0.001$ \\
\hline \multicolumn{2}{|c|}{ Platelets, $10^{3} / \mathrm{mm}^{3}$, mean $(95 \% \mathrm{CI})$} & $\begin{array}{c}240.6 \\
(223.6-257.6)\end{array}$ & $\begin{array}{c}368.6 \\
(303.7-433.6)\end{array}$ & $<0.001$ \\
\hline \multicolumn{2}{|c|}{$\begin{array}{l}\text { Time from primary to metastatic diagnosis, } \\
\text { months, median (range) }\end{array}$} & $24.7(2.2$ to 68.1$)$ & $2.5(0.0$ to 15.8$)$ & $<0.01$ \\
\hline \multicolumn{2}{|c|}{$\begin{array}{l}\text { Time from diagnosis to treatment onset, months, } \\
\text { median (range) }\end{array}$} & 28.7 (6.8 to 71.9$)$ & $3.8(1.2$ to 23.0$)$ & $<0.01$ \\
\hline \multirow[t]{2}{*}{$\begin{array}{l}\text { Metastasis at diagnosis, } \\
N(\%)\end{array}$} & No & $63(69.2)$ & $12(46.2)$ & $<0.05$ \\
\hline & Yes & $28(30.8)$ & $14(53.8)$ & \\
\hline \multicolumn{2}{|l|}{ Lung metastasis, $N(\%)$} & $59(60.8)$ & $23(88.5)$ & $<0.01$ \\
\hline \multicolumn{2}{|l|}{ Brain metastasis, $N(\%)$} & $3(3.1)$ & $4(15.4)$ & $<0.05$ \\
\hline \multicolumn{2}{|l|}{ Hepatic metastasis, $N(\%)$} & $7(7.2)$ & $6(23.1)$ & $<0.05$ \\
\hline \multirow[t]{4}{*}{ Fuhrman grade } & Grade 1 & $7(10.3)$ & $0(0)$ & $<0.05$ \\
\hline & Grade 2 & $21(30.9)$ & $4(23.5)$ & \\
\hline & Grade 3 & $30(44.1)$ & $5(29.4)$ & \\
\hline & Grade 4 & $10(14.7)$ & $8(47.1)$ & \\
\hline \multirow[t]{4}{*}{ Heng risk factors, $N(\%)$} & 0 & $22(23.7)$ & $2(8.7)$ & $<0.01$ \\
\hline & 1 & $49(52.7)$ & $7(30.4)$ & \\
\hline & 2 & $18(19.4)$ & $11(47.8)$ & \\
\hline & 3 & $4(4.3)$ & $3(13.0)$ & \\
\hline
\end{tabular}

ECOG-PS: Eastern Cooperative Oncology Group Performance Status; LR: long-term responders; PR: primary refractory; LDH: lactate dehydrogenase; CI: confidence intervals.

months (95\%CI: 55.1-71.9). In the PR cohort, median PFS and OS were 2.8 (95\%CI: 2.5-3.1) and 7.0 months (95\% CI: 3.3-10.7), respectively.

Baseline clinical characteristics that were significantly different between PR and LR patients are depicted in Table 1 . In the overall population, 94\% of patients had clear-cell histology with 7 patients having mixed histology (clear-cells plus sarcomatoid/papilar). Fourteen patients $(11 \%)$ received sunitinib after cytokines and 109 patients $(89 \%)$ in first-line, with no significant differences between groups.
Sunitinib was administered once-daily at either a starting dose of $50 \mathrm{mg}$ (93\% of patients), $37.5 \mathrm{mg}$ ( $5 \%$ ) or $25 \mathrm{mg}(2 \%)$ following 4 -weeks-on/2-weeks-off schedule. The initial dose was reduced in $73 \%$ of patients $(84 \%$ in LR group, $32 \%$ in PR group; $P<0.001$ ), mainly due to toxicity ( $92 \%$ of reductions). At the time of the study the alternative schedule 2 weeks-on/ 1 week-off was not established in clinical practice to manage toxicity.

Heng prognostic criteria were not significantly correlated with response in our population $(P=0.109)$. However, most of the components individually, except for 
calcium and neutrophil levels, showed significant differences between both cohorts, and $61 \%$ of PR patients presented $\geq 2$ Heng risk factors compared to $24 \%$ in LR group.

\section{Molecular assessment}

Fifty-two primary clear-cell primary tumor samples corresponding to $39 \mathrm{LR}(75 \%)$ and 13 PR patients (25\%) were analyzed. Among the different genes analyzed, only HES1, HEY and HEYL, putative effectors of Notch signaling, showed increased expression in the LR group compared to PR group (Figure 1A, 1B, and 1C, respectively; Figure 2). A significant positive internal correlation between the expression of these 3 genes was also observed (Figure 1D, and data not shown).

After the ROC analyses, only HEYL levels discriminated the studied populations with significant sensitivity and specificity (AUC $=0.704)$. These analyses also provided a possible cut-off value of 34.85 (reference: $G U S B)$. Of note, when the patients were classified according to their HEYL levels using this cut-off, an increased disease-specific survival in the high expression group was observed (Figure 1E).

Since HEYL is considered an effector of Notch signaling, we monitored whether LR and PR tumors displayed activated Notch. The expression of intracellular Notch domain (Notch-IC) and HEYL was determined in a TMA containing the previously analyzed samples. The samples were classified as negative or positive given their relatively small number (representative examples provided in Figure 1F). No statistically significant relationship between Notch-IC and HEYL positive staining was found $(P=0.426)$ (Figure 1F).
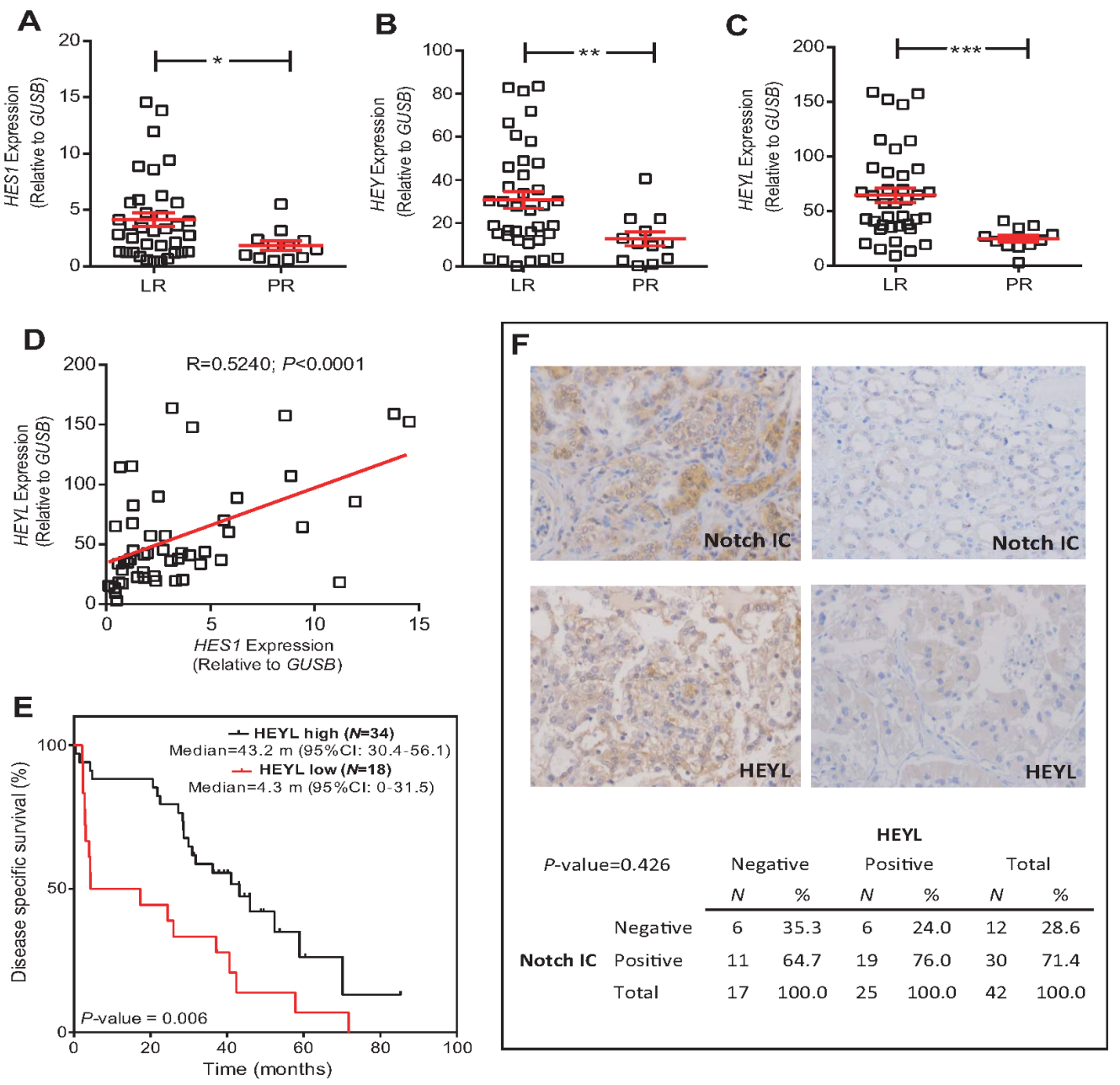

Figure 1: HEYL expression is associated with LR population. (A-C) RTqPCR analyses showing the expression of $H E S 1$ (A), $H E Y$ (B) and HEYL (C) genes in LR and PR populations. Statistical significance was obtained by Mann-Whitney's test $* P$-value $\leq 0.05$; $* * P$-value $\leq 0.01, * * * P$-value $\leq 0.005$. (D) Correlation between the HES1 and HEYL expression values. $P$-value was estimated by Pearson correlation. (E) Kaplan-Meier analysis showing that high $H E Y L$ levels were associated with increased survival ( $P$-value was obtained by the log-rank test). (F) Representative immunohistochemistry images Notch intracellular domain (NotchIC) and HEYL positive and negative tumors, and contingency tables showing the absence of significant association between NotchIC and HEYL. RTqPCR: Quantitative real-time reverse transcription polymerase chain reaction; LR: long-term responders; PR: primary refractory; CI: confidence intervals; m: months. 

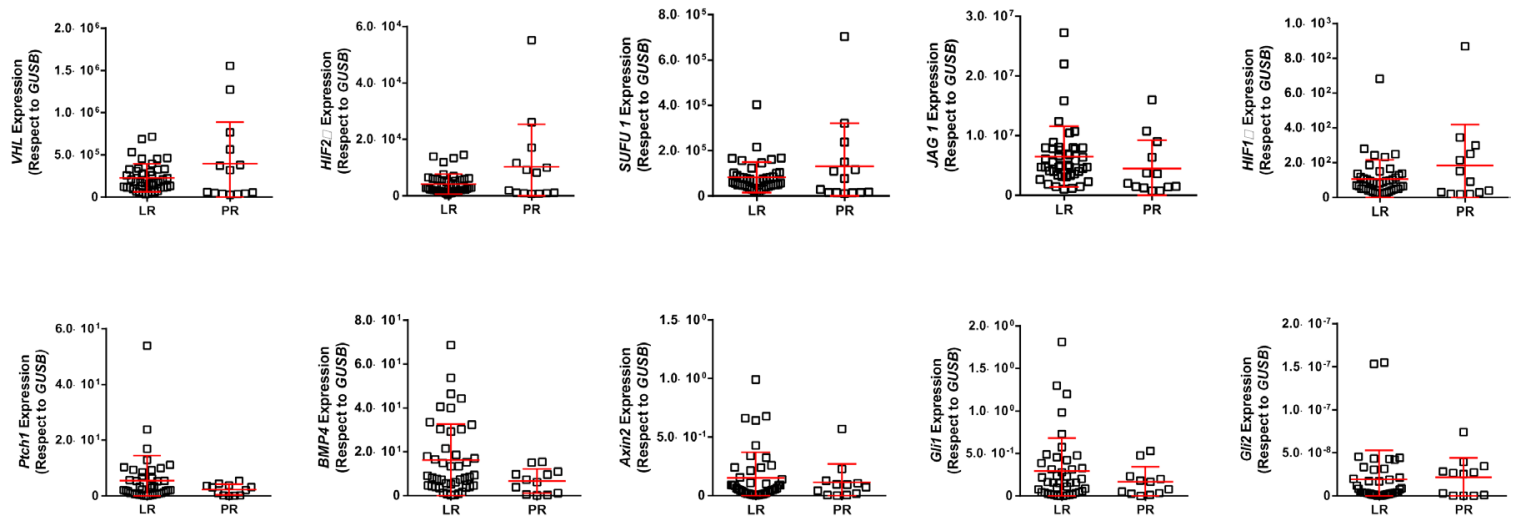

Figure 2: RTqPCR analyses showing the expression of quoted genes in LR and PR populations. RTqPCR: Quantitative real-time reverse transcription polymerase chain reaction; LR: long-term responders; PR: primary refractory.
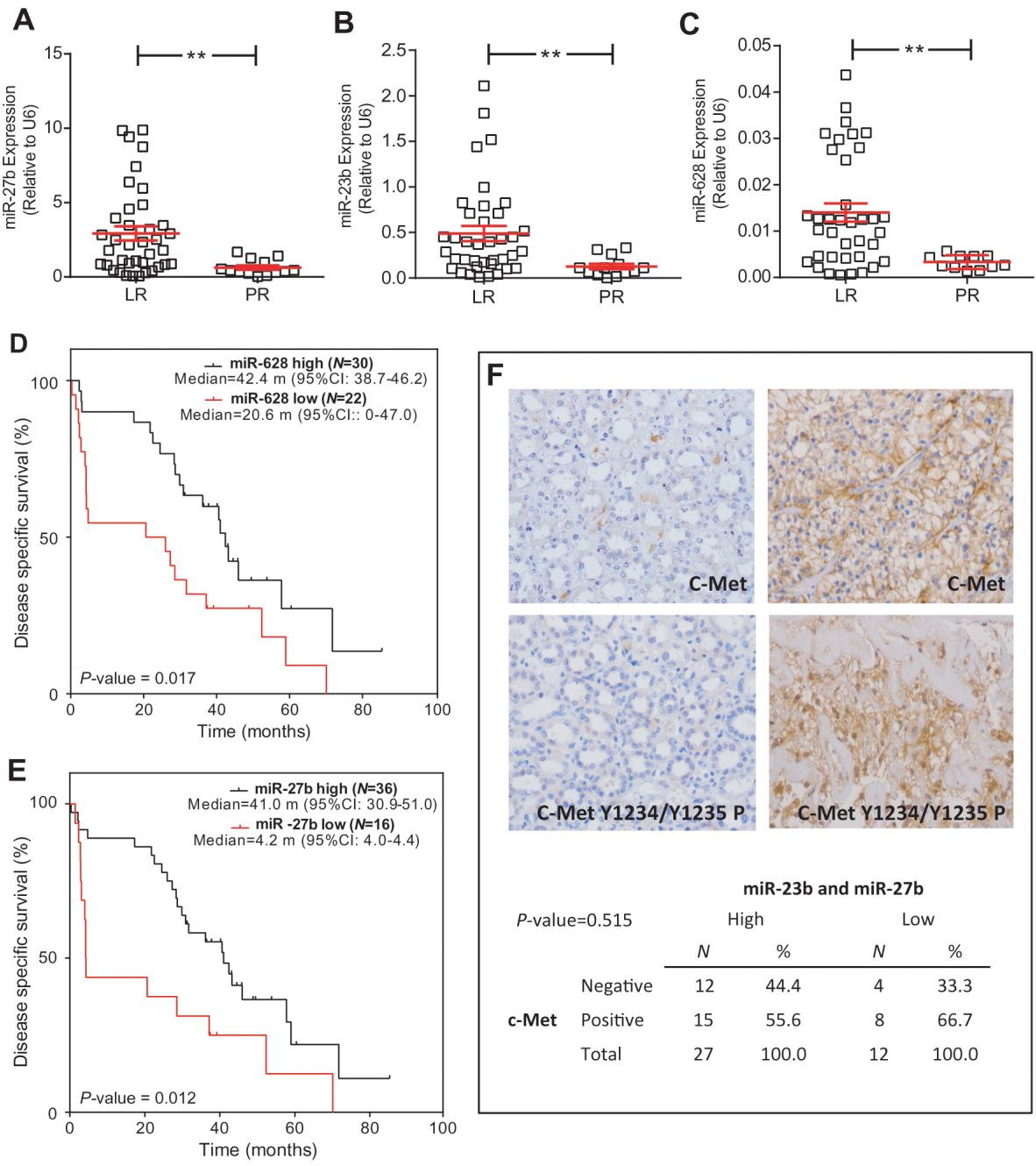

Figure 3: miRNA expression is associated with LR population. (A-C) RTqPCR analyses showing the expression of miR-23b (A), miR-27b (B) and miR-628-5p (C) in LR and PR populations. Statistical significance was obtained by Mann-Whitney's test ** $P$-value $\leq 0.01$. (D, E) Kaplan-Meier analysis showing that high levels of miR-628-5p (D) and miR-27b (E) were associated with increased survival ( $\mathrm{p}$ value was obtained by the log-rank test). (F) Representative immunohistochemistry images of positive and negative tumors for total C-Met and phosphorylated (in Tyr1234 and Tyr1235) c-Met, and contingency tables showing the absence of significant association between high levels of miR-23b and miR-27b and reduced c-Met staining. RTqPCR: Quantitative real-time reverse transcription polymerase chain reaction; LR: long-term responders; PR: primary refractory; CI: confidence intervals; m: months. 


\section{MicroRNA assessment}

Increased levels of 3 out of 13 studied miRNA were found in the LR population: hsa-miR-27b, hsa-miR-23b and hsa-miR-628-5p (Figure 3A, 3B, 3C; Figure 4). Additionally, the increased expression of these miRNAs was also associated with prolonged survival (AUC $[\mathrm{ROC}]=0.799$; 0.793 and 0.800 , respectively; cut-off values: $0.60 ; 0.15$ and 0.005 , respectively) (Figure 3D, 3E, and data not shown).

No validated targets have been yet identified for hsa-miR-628-5p. Both hsa-miR-27b and hsa-miR-23b inhibit the expression of c-Met and Notch1. A tendency of decreased c-Met activation in the LR group was observed, although statistical significance was not reached. Moreover, we did not find any association between increased levels of hsa-miR-23b or hsa-miR-27b, or the reduced levels of both, and the expression of c-Met (Figure 3F).

\section{Correlation between PFS and toxicity in LR group}

Ninety-four percent of LR patients presented at least one $\mathrm{AE}$ during the study, being the most common (all grades): asthenia (83\%), mucositis $(75 \%)$ and hand-foot syndrome $(64 \%)$, followed by hypertension, hypothyroidism, neutropenia and thrombocytopenia (57\%, 46\%, 43\% and $42 \%$, respectively). A marked tendency towards prolonged PFS was observed in patients with hypertension or hypothyroidism, but did not reach statistical significance (Figure 5).

\section{DISCUSSION}

The results of this molecular study suggest that there is a potential correlation between Notch activation and longterm response in $\mathrm{mRCC}$ patients treated with sunitinib. This correlation has been previously reported [17] and is aligned with the widely reported role of this pathway in angiogenesis $[18,19]$. Our data also support that other relevant signaling pathways, such as Hedgehog and hypoxia, are not major players in sunitinib response in $\mathrm{mRCC}$.

According to our analyses, only $H E Y L$ expression could be potentially used a biomarker of sunitinib response. Increased $H E Y L$ expression has been associated with neovascularization in breast cancer [20]. Nonetheless, other potential Notch signaling effectors, such as HES1 and $H E Y$, did not reach the statistical significance as unique biomarkers. Moreover, JAG1 levels did not discriminated studied populations, and Notch IC was not associated with increased HEYL levels. In our opinion, the low number of available tumor samples and the high intratumor heterogeneity in mCRR [21] may be limiting these results. Notch signaling is a complex process involving multiple cell compartments and proteins, and its possible role in sunitinib response requires further investigation in a larger cohort.

Various miRNAs can regulate hypoxia and angiogenesis [22], suggesting a potential role in patient's response to antiangiogenic drugs. For instance, hsamiR-221/222, hsa-miR-141, hsa-miR-942, hsa-miR133a and hsa-miR-484 have been previously suggested as candidates for poor response to sunitinib [23-26]. However, there are important discrepancies among these studies, which are also limited by the small number of patients included and their specific clinical and therapeutic response characteristics. The results herein presented, comparing extreme groups of responders to sunitinib, do not support the potential significance of these pre-specified miRNAs. Here we provide evidence that changes in hsamiR-628-5p are distinctive of LR and PR populations of sunitinib-treated mRCC patients. This miRNA was previously identified by Prior et al [25]. Nonetheless, the absence of bona fide characterized targets of hsa-miR-628$5 \mathrm{p}$ precludes ulterior functional analysis.
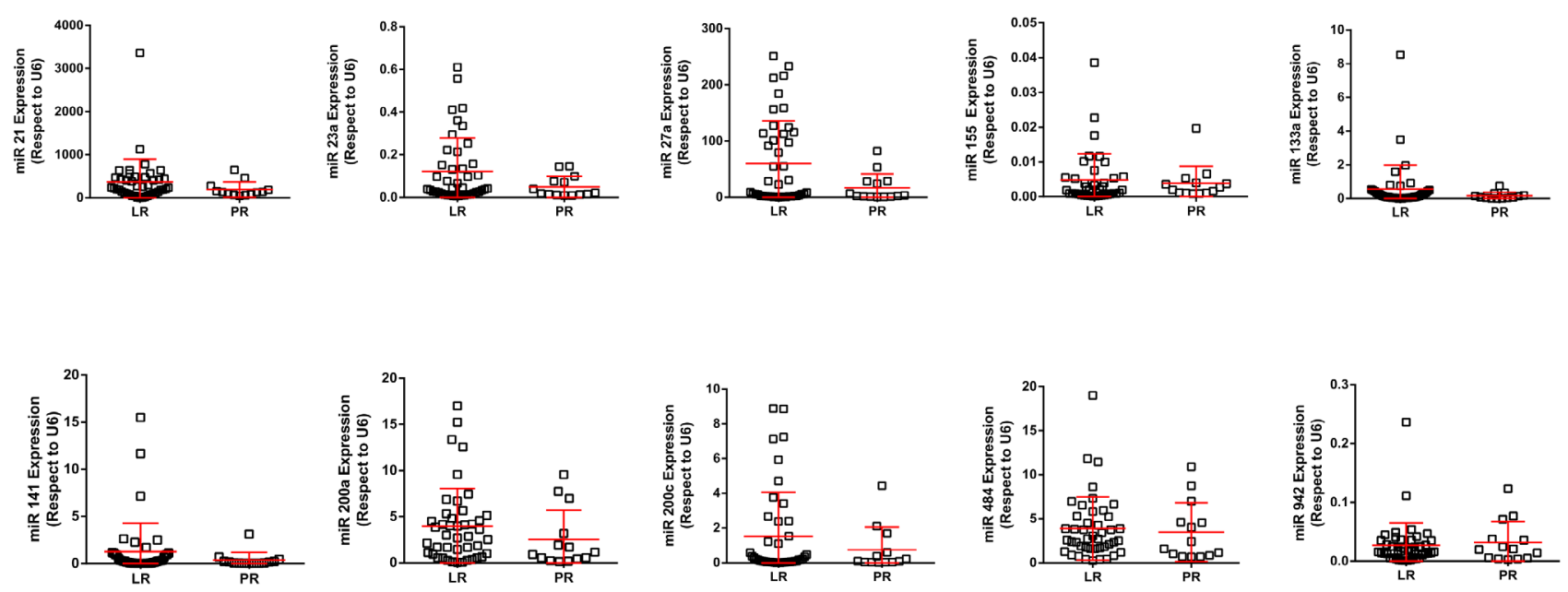

Figure 4: RTqPCR analyses showing the expression of quoted miRNAs in LR and PR populations. RTqPCR: Quantitative real-time reverse transcription polymerase chain reaction; LR: long-term responders; PR: primary refractory. 
A)

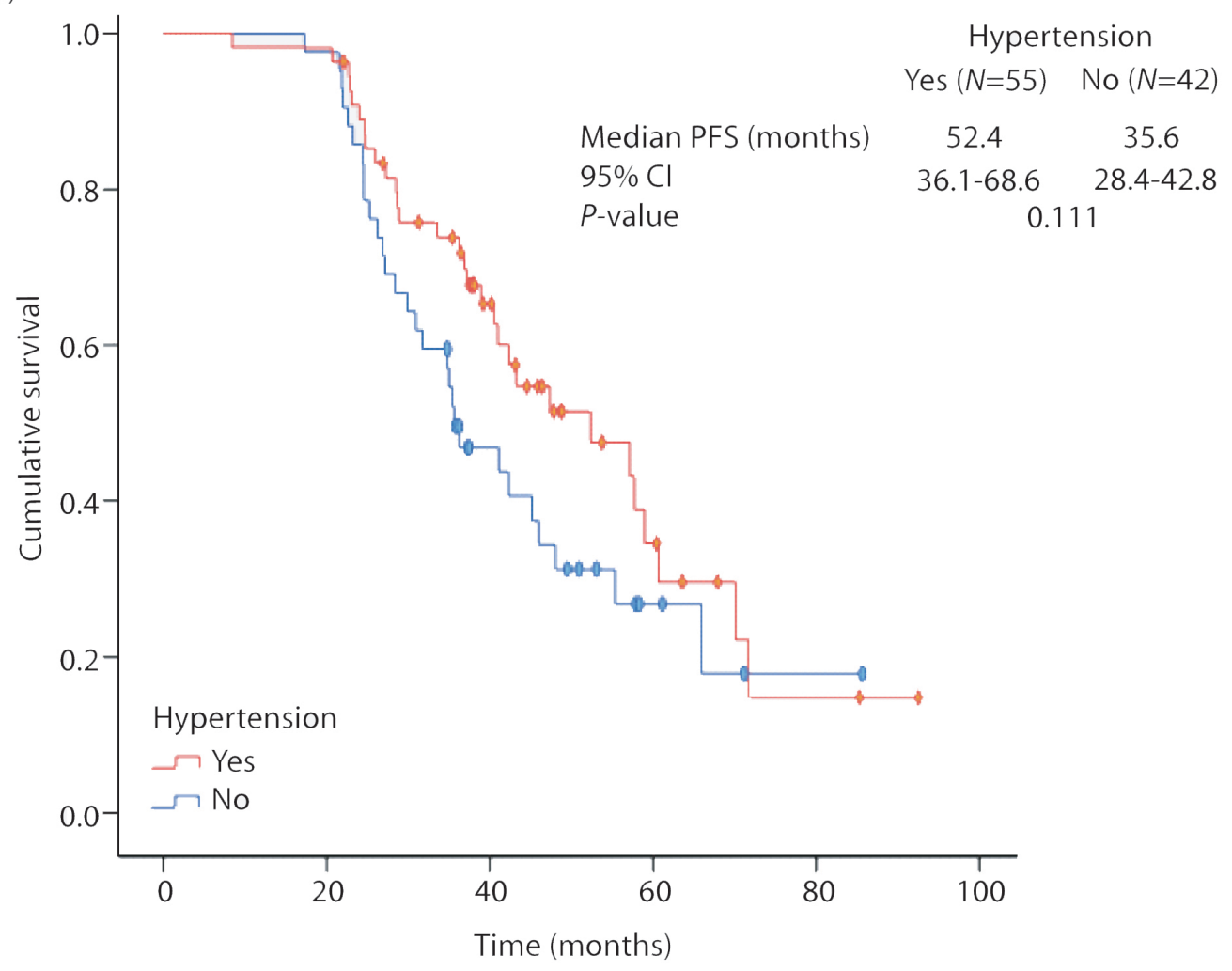

B)

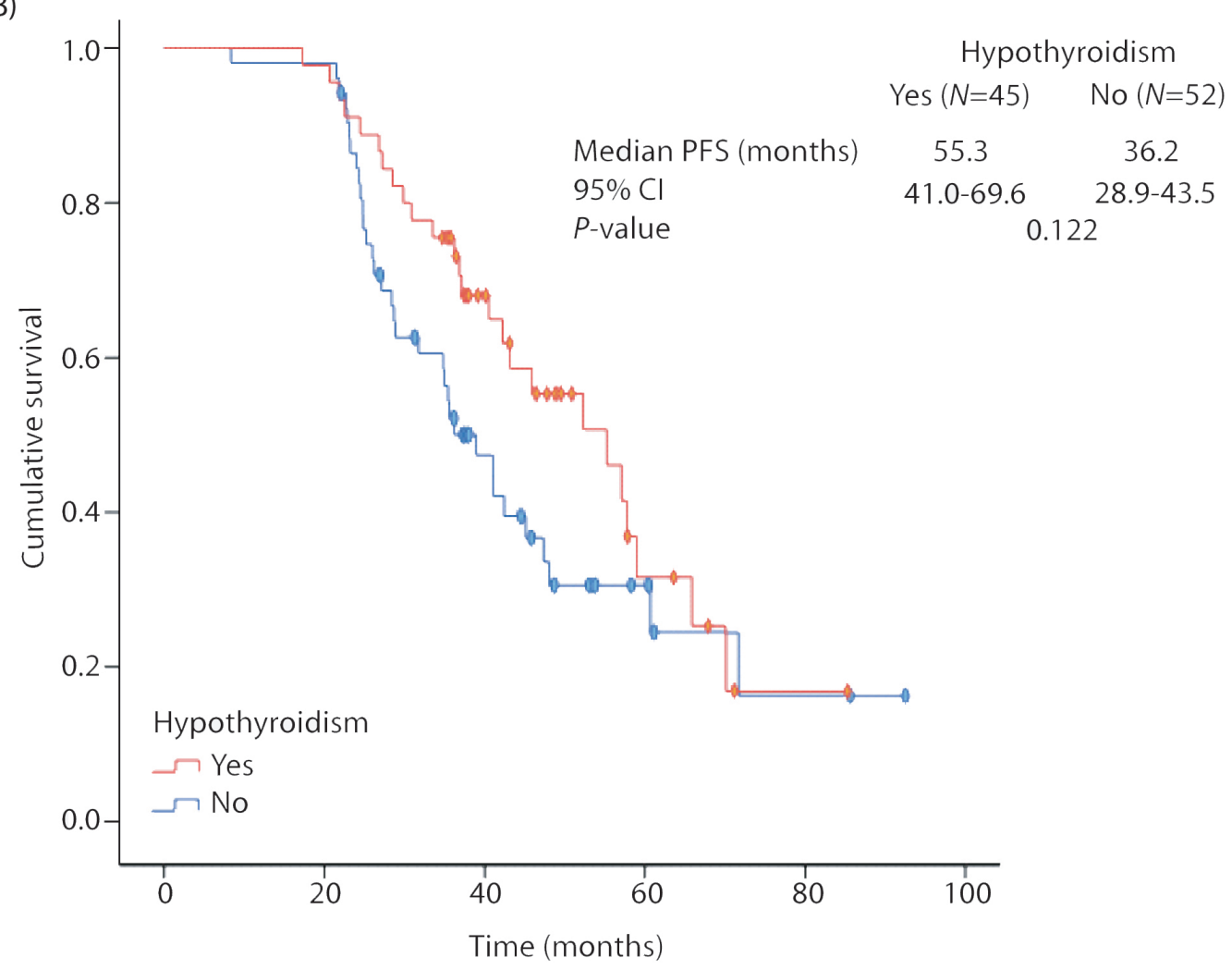

Figure 5: Progression-free survival in the long-term responders' cohort according to the occurrence of (A) hypertension; (B) hypothyroidism. Kaplan-Meier estimates. PFS: progression-free survival; CI: confidence intervals. 
Table 2: Sequences of the specific RT primer oligonucleotides used in the reverse transcription polymerase chain reaction

\begin{tabular}{|c|c|c|c|}
\hline GENE & RT & Forward & Reverse \\
\hline$H u-c M y c$ & GTTGAAGGAATCG & AATGAAAAGGCCCCCAAGGTAGTTATCC & GTCGTTTCCGCAACAAGTCCTCTTC \\
\hline$H u-A C T B$ & GCATTACATAATTTACAC & CCAACCGCGAGAAGATGA & TCCATCACGATGCCAGTG \\
\hline$H u-T B P$ & GTG TTT AAA ATC TAC ATA & AGTGAAGAACAGTCCAGACTG & CCAGGAAATAACTCTGGCTCAT \\
\hline$H u-G U S B$ & CTTCTGATACTTCTTATAC & CGCCCTGCCTATCTGTATTC & TCCCCACAGGGAGTGTGTAG \\
\hline$H u-G A P D H$ & TACTTTATTGATGGTACA & AGCCACATCGCTCAGACAC & GCCCAATACGACCAAATCC \\
\hline Hu-PPIAI & AATGGTGATTCTTCTTGCTGG & ATGCTGGACCCAACACAAAT & TCTTTCACTTTGCCAAACACC \\
\hline$H u-R P S 13$ & CTTAATTAAATGGTAGAGATC & GGTTGAAGTTGACATCTGACGA & CTTGTGCAACACCATGTGAAT \\
\hline$H u-V H L$ & CCCTGACTGACTGAAGGCT & ATCCGTAGCGGTTGGTGA & CTCACGGATGCCTCAGTCTT \\
\hline Hu-Hifla & CTGCATGATCGTC & TTTTTCAAGCAGTAGGAATTGGA & GTGATGTAGTAGCTGCATGATCG \\
\hline$H u-H i f 2$ & TAGGTGAACTTCATGTCC & TACAAGGAGCCCCTGCTGTC & TGCTGGATTGGTTCACACATG \\
\hline Hu-Gli1 & TGACTTCTGTCCCCACACTG & AGCGCCCAGACAGAGTGT & GGGGTCATCGAGTTGAACAT \\
\hline$H u-G l i 2$ & AGCTGGCTCAGCATGGTC & ACTCCACACACGCGGAAC & CCACTGAAGTTTTCCAGG \\
\hline$H u-B M P 4$ & TCCACAGCACTGGTCTTGAG & TGGGATGTTCTCCAGATGTTCT & GGGATGCTGCTGAGGTTAAA \\
\hline Hu-PTCH1 & CGAGGTTCGCTGCTTTTAAT & TCTGGAGCAGATTTCCAAGG & TTTGAATGTAACAACCCAGTTTAAATA \\
\hline Hu-HES1 & GTGCGCACCTCGGTATTAAC & GAAGCACCTCCGGAACCT & GTCACCTCGTTCATGCACTC \\
\hline$H u-H E Y$ & AGCAGATCCCTGCTTCTCAA & CGAGCTGGACGAGACCAT & GGAACCTAGAGCCGAACTCA \\
\hline$H u-H E Y L$ & GGGCATCAAAGAATCCTGTC & GTCCCCACTGCCTTTGAG & ACCGTCATCTGCAAGACCTC \\
\hline$H u-A X I N 2$ & CTTCATCCTCTCGGATCTGC & GCTGACGGATGATTCCATGT & ACTGCCCACACGATAAGGAG \\
\hline$H u-S U F U$ & ACTGCAGGGCCCA & TGTTGGAGGATTTAGAAGATTTGAC & AGGCCAGCTGTACTCTTTGG \\
\hline$H u-J A G 1$ & TTGATCATGCCCGA & GGCAACACCTTCAACCTCA & GCCTCCACAAGCAACGTATAG \\
\hline
\end{tabular}

To the best of our knowledge, this is the first report showing an association between increased levels of hsamiR-23b and hsa-miR-27b and prolonged response to sunitinib in patients with mRCC. However, their roles in other cancer types and in chemoresistance have been previously reported $[27,28]$. The joint inhibition of the expression of Notch1 and c-Met by hsa-miR-23b and hsa-miR-27b reinforces our above commented hypothesis regarding the potential involvement of Notch signaling in sunitinib response, and warrants future research on this pathway. Furthermore, MET expression has been associated with resistance to sunitinib and with more aggressive tumor behavior in renal cancer, becoming an attractive target for the treatment of sunitinib-resistant mRCC patients [29]. However, we did not find a significant association between increased levels of hsamiR-23b or hsa-miR-27b or the reduced levels of either miRNAs with the expression or activity of MET in renal cancer samples. Further studies are needed to elucidate the possible targets of these two miRNAs regarding sunitinib response in $\mathrm{mRCC}$ patients.

Additionally, despite the strong tendency observed, we did not found a significantly prolonged PFS in our cohort of LR patients presenting sunitinib-related AEs, contrary to previous studies $[6,7]$, probably due to their extremely prolonged survival time.
We observed a significantly higher number of Heng risk factors [30] in PR patients, although risk categories did not differ between groups, probably related with the low number of patients included in PR group. Additionally, we found a significantly lower proportion of nephrectomies, higher lactate dehydrogenase levels, shorter time to metastatic disease, higher presence of lung, brain and hepatic metastasis, and increased Fuhrman grade among PR patients. Some of these factors have been previously identified as predictive of a shorter PFS $[14,16]$, while others may have arose given the extreme differences of the studied populations. The validation of its prognostic value in a large independent cohort is warranted.

In conclusion, our data present important molecular implications regarding the response to sunitinib in $\mathrm{mRCC}$ patients. However, given the lack of identified molecular targets and current limitations of immunohistochemistry techniques, we were unable to identify which pathways are conditioning sunitinib response. The identification of these pathways and the confirmation of HEYL, hsamiR-27b, hsa-miR-23b and hsa-miR-628-5p as predictive biomarkers will allow clinicians to offer an accurate and personalized treatment for both newly diagnosed and nonresponder patients in daily clinical practice. 


\section{PATIENTS AND METHODS}

\section{Patients}

Eligibility criteria included adult (age $\geq 18$ years) patients with confirmed $\mathrm{mRCC}$ with a clear-cell component treated with sunitinib. Patients were classified in two groups:

- Long-term responders: patients who achieved a PFS $\geq 22$ months and at least complete response (CR), partial response or stable disease (SD).

- Primary refractory: patients who showed progressive disease in the first 3 months after sunitinib onset.

\section{Study design and assessments}

Retrospective, observational, multicenter study of two extreme groups of patients (LR and PR) treated with sunitinib under clinical practice. The study was conducted between January 2012 and January 2014 in 16 centers participating in the Spanish Oncology Geniturinary Group (SOGUG). The objectives were to identify baseline clinical factors associated with long-term clinical benefit to sunitinib treatment and to correlate tumor expression of different signaling pathways components and the occurrence of treatment-related AEs with treatment associated survival.

This study was carried out in accordance with the declaration of Helsinki and approved by the Spanish Authorities and Ethics Committees of each participating hospital. All patients gave written informed consent.

\section{Study procedures}

Immunohistochemistry and genetic analyses were carried out in available archived formalin-fixed paraffin embedded (FFPE) clear-cell primary tumor samples. Hematoxylin and eosin stained sections of the tumor samples were examined by a pathologist to confirm the diagnosis and estimated tumor content. These analyses were performed at the Molecular Laboratory of Hospital 12 de Octubre (Madrid, Spain).

The following patient characteristics and outcome data were collected using uniform data collection templates: Date of birth, gender, age at CRC diagnosis, date of first diagnosis of tumor disease, baseline TNM, baseline cancer stage, histological subtype, Furhman grade, comorbidities, nephrectomy (yes/no), nephrectomy type (total/partial), percentage of tumor necrosis, Karnofsky/ECOG performance status, baseline lactate dehydrogenase level, baseline corrected calcium level, baseline hemoglobin level, left ventricular ejection fraction, presence of thrombocytosis at baseline, presence of neutrophilia at baseline, baseline Heng risk factors, previous radiotherapy received (yes/no), number of metastatic locations, recurrence (metastatic) location and date, and treatments received (dates, type, doses, associated toxicity and response [PFS, time to progression and OS]).

\section{Quantitative real-time reverse transcription polymerase chain reaction (RT-qPCR)}

Expression levels of 13 selected miRNAs (miR942, miR-628-5p, miR-133a, miR-484, miR-141, miR23a, miR-23b, miR-27b, miR-21, miR-200a, miR-200c, miR-141and miR-155) and mRNAs (MYC, VHL, HIF1A, HIF2, GLI1, GLI2, BMP4, PTCH1, HES1, HEY, HEYL, $A X I N 2, S U F U$ and $J A G 1$ were monitored by RT-qPCR in both groups. The miRNA were selected on the basis of their involvement in angiogenesis, modulation of the Notch, Hedgehog and/or Wnt signaling pathways, or by being previously reported as biomarkers of Sunitinb response in renal cancer samples [23-26]. For the mRNA normalization six previously reported genes were assayed (ACTB, TBP, GusB, GAPDH, PPIA1 and RPS13) and evaluated using three normalizer evaluation software (GeNorm, NormFinder and BestKeeper). The best normalizer gene was $G u s B$, so the expression analyses were performed relative to this gene.

Total RNA was isolated from ten $10 \mu \mathrm{m}$ sections of each tumor sample and using miRNeasy FFPE Kit (Qiagen, Hilden, Germany) according to the manufacturer's recommendations. Reverse transcription was performed using the Omniscript RT Kit (Qiagen, Hilden, Germany), using $50 \mathrm{ng}$ of total RNA and a mix of $10 \mathrm{mM}$ of all specific RT primers (Table 2) in $50 \mu$ final volume. Polymerase chain reaction (PCR) was performed in a 7500 Fast Real Time PCR System using Go Taq PCR master mix (Promega, Fitchburg, WI, USA) and $1 \mu \mathrm{l}$ of cDNA as a template. Melting curves were performed to verify specificity and absence of primer dimers. Reaction efficiency was calculated for each primer combination. The sequences of the specific qPCR oligonucleotides are shown in Table 2. To measure quantitatively the expression of miRNAs, RNA was extracted using the same method as for the genes. Reverse transcription was carried out from $10 \mathrm{ng}$ total RNA along with miR-specific primer using the TaqMan ${ }^{\circledR}$ MicroRNA Reverse Transcription Kit (Applied Biosystems, Foster City, CA, USA). PCR assays were performed using TaqMan $^{\circledR}$ Gene Expression Master Mix and 7500 Fast Real Time PCR System (Applied Biosystems, Foster City, CA, USA) as reported [31]. For miRNA normalization, we used RNU6B. The potential genes targeted by the studied miRNA were assessed using the miRTarBase webtool (http://mirtarbase.mbc.nctu.edu.tw/) [32].

\section{Construction of tissue microarrays (TMA) and immunohistochemistry}

The expression of Notch IC (MAB3647 R\&D Systems, Minneapolis, MN, USA; 1/100 diluted), HEYL (TA324613, Origene 1/500 diluted), c-MET (AF276, 
R\&D Systems; 1/100 diluted) and c-MET phosphorylated in Y1234/Y1235 (AF2480, R\&D Systems, 1/100 diluted) were assessed by immunohistochemistry in TMA after antigen retrieval treatment. Signal was amplified using avidin-peroxidase (ABC elite kit Vector, Burlingame, CA, USA) and visualized using diaminobenzidine as a substrate (DAB kit Vector, Burlingame, CA, USA). Scoring of the results and selection of the thresholds, internal controls for reactivity of each antibody, and tissue controls for the series were done by double blind method according previously published methods [33]. At least two representative duplicate cores for each case were scored.

\section{Statistical analysis}

Protein, mRNA and miRNA expression (positive vs negative) were correlated with treatment outcome according to the cohorts of LR and PR. Survival probabilities were assessed by the Kaplan-Meier method. Cox proportional hazard models were used to identify predictors of treatment outcomes. To determine their ability to discriminate the two populations we sequentially performed unpaired tTest, ROC analyses and KaplanMeyer distributions with LogRank test. For all statistical tests, an a priori significant level of $\alpha=0.05$ was assumed.

Efficacy end points included ORR, OS and PFS, assessed by investigators using Response Evaluation Criteria in Solid Tumours v1.1 [34]. The AEs were recorded regularly and graded according to Common Terminology Criteria for Adverse Events (CTCAE) v4.0. SPSS Statistics $^{\mathcal{O}}$ software version 19 (IBM, Armonk, NY) was used for statistical analysis.

\section{Author contributions}

Javier Puente designed the research study. Javier Puente, Jesús M. Paramio, Marta Dueñas, Julián Sanz and Mónica Martínez-Fernández conducted the experiments. All listed authors acquired the data. Javier Puente, Luz Samaniego, Jesús M. Paramio, Marta Dueñas, Julián Sanz and Mónica Martínez-Fernández and performed the analysis and interpretation of data. Javier Puente supervised the study, and all authors contributed to the writing, review, and revision of the manuscript.

\section{ACKNOWLEDGMENTS}

The authors gratefully thank all contributing investigators and research teams of SOGUG group. We also thank Marina Morán and M. Victoria Bolós from Pfizer for their valuable contribution in all the steps of the study. The authors also appreciate the collaboration of the participating patients and their families. Medical Writing: TFS: Juan Martín; Financial Support: Pfizer S.L.U.

\section{CONFLICTS OF INTEREST}

Javier Puente has received honoraria for advisory boards by Astellas, Bayer, Janssen, Novartis, Pfizer, Roche and Sanofi; Nuria Laínez has received honoraria for advisory boards by Pfizer; Luis León has received honoraria for advisory boards by Astellas, Bayer, GSK, Janssen, Novartis, Pfizer, Roche and Sanofi; Julio Lambea has received honoraria for advisory boards and travel expenses by Astellas, Bayer, Janssen, Novartis, Pfizer and Sanofi; Begoña Pérez-Valderrama has received honoraria for advisory boards by Astellas, Bayer, BMS, Pierre-Fabre and Roche, for travel expenses by Janssen and for lectures by Bayer, Novartis and Pierre-Fabre; Sergio Vázquez has received honoraria for advisory boards and travel expenses by Pfizer; Enrique Gallardo has received honoraria for advisory boards and travel expenses by Bayer, BMS, Novartis and Pfizer, and for lectures by Pfizer; José Pablo Maroto has received honoraria for advisory boards by Astellas, Bayer, Janssen, Novartis, Pfizer, Roche and Sanofi. All remaining authors have declared no conflicts of interest.

\section{FUNDING}

This study was supported by an unrestricted grant from Pfizer (SOGUG-2011-05), and partially by Spanish Ministerio de Economía, Industria y Competitividad (MINECO) and European Fondo Europeo de Desarrollo Regional (FEDER) grants SAF2015-66015-R, ISCIII-RETIC RD12/0036/0009, PIE 15/00076, and CB16/12/00228 granted to Jesús M. Paramio.

\section{REFERENCES}

1. Vermassen T, De Meulenaere A, Van de Walle M, Rottey $\mathrm{S}$. Therapeutic approaches in clear cell and non-clear cell renal cell carcinoma. Acta Clin Belg. 2016: 1-7. doi: 10.1080/17843286.2016.1193269.

2. Motzer RJ, Hutson TE, Tomczak P, Michaelson MD, Bukowski RM, Oudard S, Negrier S, Szczylik C, Pili R, Bjarnason GA, Garcia-del-Muro X, Sosman JA, Solska $\mathrm{E}$, et al. Overall survival and updated results for sunitinib compared with interferon alfa in patients with metastatic renal cell carcinoma. J Clin Oncol. 2009; 27: 3584-3590. doi: 10.1200/JCO.2008.20.1293.

3. Garcia-Donas J, Esteban E, Leandro-García LJ, Castellano DE, del Alba AG, Climent MA, Arranz JA, Gallardo E, Puente J, Bellmunt J, Mellado B, Martínez E, Moreno F, et al. Single nucleotide polymorphism associations with response and toxic effects in patients with advanced renalcell carcinoma treated with first-line sunitinib: a multicentre, observational, prospective study. Lancet Oncol. 2011; 12: 1143-1150. doi: 10.1016/S1470-2045(11)70266-2.

4. Xu CF, Bing NX, Ball HA, Rajagopalan D, Sternberg CN, Hutson TE, de Souza P, Xue ZG, McCann L, King KS, Ragone LJ, Whittaker JC, Spraggs CF, et al. Pazopanib 
efficacy in renal cell carcinoma: evidence for predictive genetic markers in angiogenesis-related and exposurerelated genes. J Clin Oncol. 2011; 29: 2557-2264. doi: 10.1200/JCO.2010.32.9110.

5. Muriel López C, Esteban E, Astudillo A, Pardo P, Berros JP, Izquierdo M, Crespo G, Fonseca PJ, Sanmamed M, Martínez-Camblor P. Predictive factors for response to treatment in patients with advanced renal cell carcinoma. Invest New Drugs. 2012; 30: 2443-2449. doi: 10.1007/ s10637-012-9836-4.

6. Rini BI, Cohen DP, Lu DR, Chen I, Hariharan S, Gore ME, Figlin RA, Baum MS, Motzer RJ. Hypertension as a biomarker of efficacy in patients with metastatic renal cell carcinoma treated with sunitinib. J Natl Cancer Inst. 2011; 103: 763-773. doi: 10.1093/jnci/djr128.

7. Donskov F, Michaelson MD, Puzanov I, Davis MP, Bjarnason GA, Motzer RJ, Goldstein D, Lin X, Cohen DP, Wiltshire R, Rini BI. Sunitinib-associated hypertension and neutropenia as efficacy biomarkers in metastatic renal cell carcinoma patients. Br J Cancer. 2015; 113: 1571-1580. doi: 10.1038/bjc.2015.368.

8. Alketbi A, Attoub S. Notch Signaling in Cancer: Rationale and Strategies for Targeting. Curr Cancer Drug Targets. 2015; 15: 364-374.

9. Mooney CJ, Hakimjavadi R, Fitzpatrick E, Kennedy E, Walls D, Morrow D, Redmond EM, Cahill PA. Hedgehog and Resident Vascular Stem Cell Fate. Stem Cells Int. 2015; 2015: 468428. doi: 10.1155/2015/468428.

10. Li J, Ji L, Chen J, Zhang W, Ye Z. Wnt/ $\beta$-Catenin Signaling Pathway in Skin Carcinogenesis and Therapy. BioMed Res Int. 2015; 2015: 964842. doi: 10.1155/2015/964842.

11. Chen HF, Wu KJ. Endothelial Transdifferentiation of Tumor Cells Triggered by the Twist1-Jagged1-KLF4 Axis: Relationship between Cancer Stemness and Angiogenesis. Stem Cells Int. 2016; 2016: 6439864. doi: 10.1155/2016/6439864.

12. Yan XC, Cao J, Liang L, Wang L, Gao F, Yang ZY, Duan JL, Chang TF, Deng SM, Liu Y, Dou GR, Zhang J, Zheng QJ, et al. miR-342-5p Is a Notch Downstream Molecule and Regulates Multiple Angiogenic Pathways Including Notch, Vascular Endothelial Growth Factor and Transforming Growth Factor $\beta$ Signaling. J Am Heart Assoc. 2016; 5: e003042. doi: 10.1161/JAHA.115.003042.

13. Heinrich EM, Dimmeler S. MicroRNAs and stem cells: control of pluripotency, reprogramming, and lineage commitment. Circ Res. 2012; 110: 1014-1022. doi: 10.1161/CIRCRESAHA.111.243394.

14. Molina AM, Jia X, Feldman DR, Hsieh JJ, Ginsberg MS, Velasco S, Patil S, Motzer RJ. Long-term response to sunitinib therapy for metastatic renal cell carcinoma. Clin Genitourin Cancer. 2013; 11: 297-302. doi: 10.1016/j. clgc.2013.04.001.

15. Porta C, Gore ME, Rini BI, Escudier B, Hariharan S, Charles LP, Yang L, DeAnnuntis L, Motzer RJ.
Long-term Safety of Sunitinib in Metastatic Renal Cell Carcinoma. Eur Urol. 2016; 69: 345-351. doi: 10.1016/j. eururo.2015.07.006.

16. Motzer RJ, Escudier B, Bukowski R, Rini BI, Hutson TE, Barrios CH, Lin X, Fly K, Matczak E, Gore ME. Prognostic factors for survival in 1059 patients treated with sunitinib for metastatic renal cell carcinoma. Br J Cancer. 2013; 108: 2470-2477. doi: 10.1038/bjc.2013.236.

17. Dai H, Fishman MN, Ching KA, Williams JA, Teer JK, English PA, Zhang Y, Murray BW, Kumar N, Huntsman S, Berglund AE, Dalton WS, Matczak E, et al. Identification of tumor biomarkers for sunitinib in advanced renal cell carcinoma (RCC). J Clin Oncol. 2015; 33: 4106-11. doi: 10.1200/JCO.2015.63.7918.

18. Hori K, Sen A, Artavanis-Tsakonas S. Notch signaling at a glance. J Cell Sci. 2013; 126: 2135-2140. doi: 10.1242/ jes. 127308.

19. Kerbel RS. Tumor Angiogenesis. N Engl J Med. 2008; 358 : 2039-2049. doi: 10.1056/NEJMra0706596.

20. Parker BS, Argani P, Cook BP, Liangfeng H, Chartrand SD, Zhang M, Saha S, Bardelli A, Jiang Y, St Martin TB, Nacht M, Teicher BA, Klinger KW, et al. Alterations in vascular gene expression in invasive breast carcinoma. Cancer Res. 2004; 64: 7857-7866. doi: 10.1158/0008-5472. CAN-04-1976.

21. Gerlinger M, Rowan AJ, Horswell S, Larkin J, Endesfelder D, Gronroos E, Martinez P, Matthews N, Stewart A, Tarpey $\mathrm{P}$, Varela I, Phillimore B, Begum S, et al. Intratumor heterogeneity and branched evolution revealed by multiregion sequencing. N Engl J Med. 2012; 366: 883892. doi: 10.1056/NEJMoa1113205.

22. Suárez Y, Sessa WC. MicroRNAs as novel regulators of angiogenesis. Circ Res. 2009; 104: 442-454. doi: 10.1161/ CIRCRESAHA.108.191270.

23. Khella HWZ, Butz H, Ding Q, Rotondo F, Evans KR, Kupchak P, Dharsee M, Latif A, Pasic MD, Lianidou E, Bjarnason GA, Yousef GM. miR-221/222 Are Involved in Response to Sunitinib Treatment in Metastatic Renal Cell Carcinoma. Mol Ther. 2015; 23: 1748-1758. doi: 10.1038/ mt.2015.129.

24. Berkers J, Govaere O, Wolter P, Beuselinck B, Schöffski P, van Kempen LC, Albersen M, Van den Oord J, Roskams T, Swinnen J, Joniau S, Van Poppel H, Lerut E. A possible role for microRNA-141 down-regulation in sunitinib resistant metastatic clear cell renal cell carcinoma through induction of epithelial-to-mesenchymal transition and hypoxia resistance. J Urol. 2013; 189: 1930-1938. doi: 10.1016/j. juro.2012.11.133.

25. Prior C, Perez-Gracia JL, Garcia-Donas J, RodriguezAntona C, Guruceaga E, Esteban E, Suarez C, Castellano D, del Alba AG, Lozano MD, Carles J, Climent MA, Arranz $\mathrm{JA}$, et al. Identification of tissue microRNAs predictive of sunitinib activity in patients with metastatic renal cell 
carcinoma. PloS One. 2014; 9: e86263. doi: 10.1371/ journal.pone.0086263.

26. García-Donas J, Beuselinck B, Inglada-Pérez L, Graña O, Schöffski P, Wozniak A, Bechter O, Apellániz-Ruiz M, Leandro-García LJ, Esteban E, Castellano DE, González del Alba A, Climent MA, et al. Deep sequencing reveals microRNAs predictive of antiangiogenic drug response. JCI Insight. 2016; 1: e86051. doi: 10.1172/jci.insight.86051.

27. Viswanathan V, Fields J, Boman BM. The miRNA23bregulated signaling network as a key to cancer development-implications for translational research and therapeutics. J Mol Med (Berl). 2014; 92: 1129-1138. doi: 10.1007/s00109-014-1208-4.

28. Shang Y, Feng B, Zhou L, Ren G, Zhang Z, Fan X, Sun Y, Luo G, Liang J, Wu K, Nie Y, Fan D. The miR27b-CCNG1P53-miR-508-5p axis regulates multidrug resistance of gastric cancer. Oncotarget. 2016; 7: 538-549. doi: 10.18632/oncotarget.6374.

29. Zhou L, Liu XD, Sun M, Zhang X, German P, Bai S, Ding Z, Tannir N, Wood CG, Matin SF, Karam JA, Tamboli P, Sircar $\mathrm{K}$, et al. Targeting MET and AXL overcomes resistance to sunitinib therapy in renal cell carcinoma. Oncogene. 2016; 35: 2687-2697. doi: 10.1038/onc.2015.343.

30. Heng DY, Mackenzie MJ, Vaishampayan UN, Bjarnason GA, Knox JJ, Tan MH, Wood L, Wang Y, Kollmannsberger C, North S, Donskov F, Rini BI, Choueiri TK. Primary anti-vascular endothelial growth factor (VEGF)-refractory metastatic renal cell carcinoma: clinical characteristics, risk factors, and subsequent therapy. Ann Oncol. 2012; 23 : 1549-1555. doi: 10.1093/annonc/mdr533.

31. Martínez-Fernández M, Dueñas M, Feber A, Segovia C, García-Escudero R, Rubio C, López-Calderón FF, DíazGarcía C, Villacampa F, Duarte J, Gómez-Rodriguez MJ, Castellano D, Rodriguez-Peralto JL, et al. A Polycombmir200 loop regulates clinical outcome in bladder cancer. Oncotarget. 2015; 6: 42258-42275. doi: 10.18632/ oncotarget.5546.

32. Chou CH, Chang NW, Shrestha S, Hsu SD, Lin YL, Lee WH, Yang CD, Hong HC, Wei TY, Tu SJ, Tsai TR, Ho SY, Jian TY, et al. miRTarBase 2016: updates to the experimentally validated miRNA-target interactions database. Nucleic Acids Res. 2016; 44: D239-247. doi: 10.1093/nar/gkv1258.

33. Dueñas $M$, Martínez-Fernández $M$, García-Escudero R, Villacampa F, Marqués M, Saiz-Ladera C, Duarte J, Martínez V, Gómez MJ, Martín ML, Fernández M, Castellano D, Real FX, et al. PIK3CA gene alterations in bladder cancer are frequent and associate with reduced recurrence in non-muscle invasive tumors. Mol Carcinog. 2015; 54: 566-576. doi: 10.1002/mc.22125.

34. Eisenhauer EA, Therasse P, Bogaerts J, Schwartz LH, Sargent D, Ford R, Dancey J, Arbuck S, Gwyther S, Mooney M, Rubinstein L, Shankar L, Dodd L, et al. New response evaluation criteria in solid tumours: revised RECIST guideline (version 1.1). Eur J Cancer. 2009; 45: 228-247. doi: 10.1016/j.ejca.2008.10.026. 